\title{
INFLUENCE OF ELECTRODE DISTANCE ON HEATING BEHAVIOUR ASSOCIATED TO RADIO FREQUENCY PROCESSING OF LOW MOISTURE FOODS
}

\author{
H.J. SHI ${ }^{a *}$, Z.J. SuN , Z.M. YAN ${ }^{b}$ and J.B. ReN ${ }^{a}$ \\ ${ }^{\mathrm{a} C o l l e g e}$ of Mechanical and Electronic Engineering, Fujian Agriculture and Forestry University, No.15 \\ Shangxiadian Road, Cangshan District, 350002 Fuzhou. China \\ ${ }^{\mathrm{b}}$ College of Food Science and Technology, Fujian Agriculture and Forestry University, No.15 Shangxiadian Road, \\ Cangshan District, 350002 Fuzhou. China
}

(Received: 8 February 2017; accepted: 11 May 2017)

Temperature uniformity and heating rate subjected to radio frequency (RF) heating have major impact on the quality of treated low moisture foods. The objective of this paper was to analyse the influence of electrode distance on the heating behaviour of RF on condition that the sample shape, size, and location between the electrodes were defined. Considering peanut butter (PB) and wheat flour (WF) as sample food, a 3D computer simulation model was developed using COMSOL, which was experimentally validated by a RF machine (27.12 MHz, $6 \mathrm{~kW})$. Specifically, the electrode distances were selected as 84, 89, 93, 99 and 89, 93, 98, $103(\mathrm{~mm})$ for RF heating of PB and WF, respectively. Results showed that the simulated results and experimental data agreed well; the temperature-time histories of the RF heating of PB and WF were approximate straight lines; both the temperature uniformity index and the heating rate decreased with the increase of the electrode distance; the heating rate had a negative logarithmic linear relationship with the electrode distance, which was independent of the types, geometry shapes and sizes of low moisture foods.

Keywords: radio frequency heating, computer simulation, temperature uniformity, heating rate

Radio frequency (RF) refers to the electromagnetic wave ranging between $3 \mathrm{kHz} \sim 300 \mathrm{MHz}$. As a high-frequency electromagnet wave, RF is characterised by quick temperature rise and low energy consumption. Compared to microwave system, RF system is superior due to easier controllable energy field, higher stability of heating mode, larger depth of penetration, and lower investment cost. Therefore, it possesses promising potentials in thermal treatment of materials that have poor thermal conductivity and heat sensitivity, such as drying of agricultural products, deinsectization, and food sterilization, especially for foods in powder state and with high porosity and low moisture content (OzTURK et al., 2016).

By RF-based thermal treatment of agricultural products and foods, heating uniformity and heating rate are two important indices closely related with product quality. It is widely accepted that heating uniformity and heating rate can be influenced by various factors, such as dielectric property, shape and size of dielectric materials, relative position to electrodes, as well as electrode structure and voltage of the RF system. Nevertheless, existing researches on heating uniformity mainly concentrate on RF deinsectization of harvested agricultural products and effects of RF system configuration and parameters on heating uniformity, such as wheat and corn seeds (JiAO et al., 2016a) and coffee bean (PAN et al., 2012) processing with RF-hot wind, shell eggs processing with RF-hot water (GEVEKE et al., 2017), intermittent

\footnotetext{
* To whom correspondence should be addressed.

Phone: +86-13385918020; e-mail:shj_fjau@163.com
} 
heating of walnuts with multiple continuous RF incentive units (WANG et al., 2005). In the RF sterilization field of foods, existing researches have discussed roasted peanuts processing with RF-hot wind (JIAO et al., 2016b), PEI-assisted RF heating of bagged peanut butter (JIAO et al., 2014a; SHI et al., 2016), etc. In addition, some researchers have studied heating uniformity of RF on alternative agricultural products and foods, such as $1 \%$ sodium carboxymethyl cellulose solution (WANG et al., 2008) and polyurethane foam (WANG et al., 2014).

Only few researches on heating rate of RF in agricultural products and foods have been reported yet. Also, they mainly focus on the relationship between dielectric coefficient and heating rate (JIAO et al., 2014b; WANG et al., 2007; WANG et al., 2013). There are no comprehensive and systematic studies on the relationships between various geometric / physical parameters and heating uniformity / rate of the RF heating system. Specific influences of single parameters on heating uniformity and heating rate under specific conditions still remain unknown. As there is an important relationship between electrode distance and heating uniformity and heating rate in the RF system, influence of the electrode distance on heating uniformity and heating rate of the RF system in a high-protein, oily thick material, peanut butter (PB), and powder material, wheat flour (WF), were discussed in this paper.

\section{Materials and methods}

\subsection{Materials}

PB (Yinger brand, Shandong Yinger Food Co. Ltd., Zaozhuang, China) and WF (Guchuan brand, Beijing Guchuan Food Co. Ltd., Beijing, China) were purchased from a local supermarket (Yonghui, Fuzhou, China). PB is a homogeneous paste without peanut chunks and oil separation. WF is all-purpose flour that does not contain any food additives.

\subsection{Experimental apparatus and research methods}

1.2.1. Experimental apparatus. The free oscillation system (Model SO6B, Monga Strayfield, United Kingdom) with $6 \mathrm{~kW}$ rated power and $27.12 \mathrm{MHz}$ frequency was used as the RF heating system. It consists of a RF wave generator, a heating chamber (including adjustable upper and bottom parallel electrodes), and an online fibre optic monitor of temperature (Fotsdina-2080-NS/N 1448C001, Indigo Precision, Canada). More information on the RF system can be found by BOREDDY and co-workers (2014) and KONG and co-workers (2016).

1.2.2. Research methods. In RF heating, materials are put in a round polypropylene plastic box (inner diameter: $50 \mathrm{~mm}$; height: $50 \mathrm{~mm}$; wall thickness: $1 \mathrm{~mm}$ ) which will be placed onto the centre of the bottom electrode. Temperatures at monitoring points (physical centre of materials) were collected in time by the optical temperature sensor. The material was put under the infrared thermal imaging camera (IRI 4010 Multi-Purpose Imager, Irisys, Northampton, UK) immediately after the RF heating to collect surface temperature. Each thermal image carries information of temperature at different surface points of materials. The contour map of surface temperature was analysed by the data analysis software Sigmaplot (v12, Systat Software Inc.).

In existing studies, main temperature measurements include thermocouple thermometer, single-point temperature measurement with fibre optic temperature sensor, and infrared 
thermal imaging surface temperature measurement. However, it is difficult to gain temperatures at different points of three-dimensional model of materials. Considering limitations in temperature measurement, numerical analysis has specific significance to study RF heating. Real-time collection of material temperature and electromagnetic field distribution in RF heating chamber can be realized with the numerical model. Previous researches also have proved the feasibility of numerical analysis in RF heating of agricultural products and foods (Chan et al., 2004; TiWARI et al., 2011; SHI et al., 2016; UYAR et al., 2016). In this paper, RF heating uniformity was analysed by electromagnetic field and temperature field coupling computation model with experimental verification. Specifically, the colour cloud chart and contour map of surface temperature in numerical analysis were compared with the infrared thermal image and contour map in physical test. Meanwhile, results were evaluated by combining numerical analysis and heating rate at monitoring points. Then, the numerical model in accordance with these three results was used to analyse electric field distribution, temperature distribution, and uniformity.

\subsection{Numerical simulation model}

The RF heating process is divided into two parts: the conversion of electromagnetic energy to thermal energy and the process of heat transfer. Independent numerical simulation of these two processes is mainly accomplished by solving the Maxwell's equation and the heat transfer equations.

1.3.1. Theory of RF electromagnetic heating. To determine power density and temperature of isotropic, linear, and homogeneous materials, the electric field distribution can be calculated from the Maxwell curl equation (CHAN et al., 2004). Since the electrode distance of the RF system is far shorter than its wavelength (the RF wavelength of 27.12 $\mathrm{MHz}$ is about $11 \mathrm{~m}$ ), the Maxwell curl equation can be solved by quasi-static method (Metaxas, 1996). This equals the Laplace's equation:

$$
\nabla \cdot\left[\left(\sigma+\mathrm{j} \cdot 2 \pi f \varepsilon_{0} \varepsilon^{\prime}\right) \nabla U\right]=0
$$

where $\sigma$ is the conductivity, $\mathrm{j}=\sqrt{-1}, f$ is the frequency, $\varepsilon_{0}$ is the permittivity of vacuum, $\varepsilon^{\prime}$ is the dielectric constant of materials, and $U$ is the electromotive force ( $\nabla U=-E$, where $E$ is the electric field intensity). International standard units were applied to all physical variables.

1.3.2. Heat transfer theory of RF heating. RF heating of agricultural products and foods meets the equation of heat conduction:

$$
\rho C_{\mathrm{P}} \frac{\partial T}{\partial t}=\nabla \cdot(k \nabla T)+Q
$$

where $\rho$ is the density of the material $\left(\mathrm{kg} \cdot \mathrm{m}^{-3}\right), C_{\mathrm{p}}$ is the specific heat capacity $\left(\mathrm{J} \cdot \mathrm{kg}^{-1} \cdot \mathrm{K}^{-1}\right), T$ is the temperature $(\mathrm{K}), t$ is the time $(\mathrm{s})$, and $k$ is the thermal conductivity $\left(\mathrm{W} \cdot \mathrm{m}^{-1} \cdot \mathrm{K}^{-1}\right) . Q$ is the power density of heat source $\left(\mathrm{W} \cdot \mathrm{m}^{-3}\right)$, which is the heat energy transferred from electromagnetic energy of RF and can be calculated by (CHOI \& KONRAD, 1991):

$$
Q=2 \pi f \varepsilon_{0} \varepsilon^{\prime \prime} E^{2}
$$

where $\varepsilon^{\prime \prime}$ is dielectric loss factor of materials. 
1.3.3. Solving method. The COMSOL Mutliphysics based on finite element theory is a multi-physics coupling analysis software and can solve Equations 1-3 simultaneously. In this paper, the numerical simulation model of RF heating was established by COMSOL Mutliphysics. The working machine configurations of the model were i7-2600 CPU, $3.4 \mathrm{GHz}$ quad-core processor, 16 GB memory, and 64-bit Windows 7 operating system.

1.3.4. Initial and boundary conditions. The furnace wall of heating chamber was set to electric insulation $\bullet \nabla E=0$ and thermal insulation $\nabla T=0$. The bottom electrode voltage was 0 , while the upper electrode voltage was set according to different test conditions (Table 1). Initial temperature and environment temperature were determined according to corresponding test temperature, ranging between $20-25^{\circ} \mathrm{C}$ (Table 1). Size of the heating chamber, samples, and material boxes as well as dielectric properties, conductivity coefficient, and specific heat capacity of the numerical simulation model of PB and WF were introduced in the works of JIAO and co-workers (2014a) and TIWARI co-workers (2011).

1.3.5. Uniformity evaluation index. No agreed evaluation index of RF heating uniformity is available. Here, the uniformity evaluation index in Equation 4 was used, which proved more reasonable than other uniformity evaluation indices (JiAO et al., 2015).

$$
U I=\frac{\int_{V_{v o l}}\left|T-T_{t}\right| d V_{v o l}}{\left(T_{t}-T_{\text {initial }}\right) V_{\text {vol }}}
$$

where $U I$ (Uniformity Index) is the temperature uniformity index. Smaller UI indicates better heating uniformity. $T$ is temperature at any point of the material in the numerical simulation model, $T_{\mathrm{t}}$ is the targeting temperature of RF heating, $T_{\text {initial }}$ is the initial temperature, and $V_{v o l}$ is volume of the object $\left(\mathrm{m}^{3}\right)$.

Table 1. Initial and boundary condition and uniformity indices for simulated model of peanut butter (PB) and wheat flour (WF)

\begin{tabular}{lllll|llll}
\hline Major parameters & \multicolumn{3}{c}{ PB } & \multicolumn{5}{c}{ WF } \\
\hline Electrode distance, $\mathrm{mm}$ & $\mathrm{d} 84$ & $\mathrm{~d} 89$ & $\mathrm{~d} 93$ & $\mathrm{~d} 99$ & $\mathrm{~d} 89$ & $\mathrm{~d} 93$ & $\mathrm{~d} 98$ & $\mathrm{~d} 103$ \\
Initial temperature, $\mathrm{K}$ & 295.55 & 293.65 & 295.65 & 295.65 & 295.15 & 294.15 & 293.65 & 293.65 \\
Electrode voltage, $\mathrm{V}$ & 14800 & 11800 & 11200 & 10000 & 8200 & 7400 & 6800 & 5800 \\
Heating time, $\mathrm{s}$ & 220 & 230 & 230 & 230 & 80 & 110 & 157 & 230 \\
Uniformity index & 0.289 & 0.281 & 0.223 & 0.194 & 0.341 & 0.330 & 0.319 & 0.295 \\
\hline
\end{tabular}

\section{Results and discussion}

\subsection{Modelling results and discussion}

Simulated and experimental temperature contours on the top surface of PB and WF treated by RF are shown in Figure 1. Limited by article length, the RF heating of PB only gives the map of distance between $\mathrm{d} 99$ electrodes, WF only gives the map of d103. Comparison of simulated and experimental results of time-temperature history in the physical center point of PB are shown in Figure 2, while results for WF are shown in Figure 3. It can be seen from the colour cloud charts and contour maps of surface temperature of the PB and WF that experimental and simulated temperature at the physical centre agree mutually, thus proving the validity of the established numerical simulation model. The time-temperature history 
curve of the treated sample is approximately a straight line, which is in accordance with the time-temperature history curve of other agricultural products and foods in RF heating.

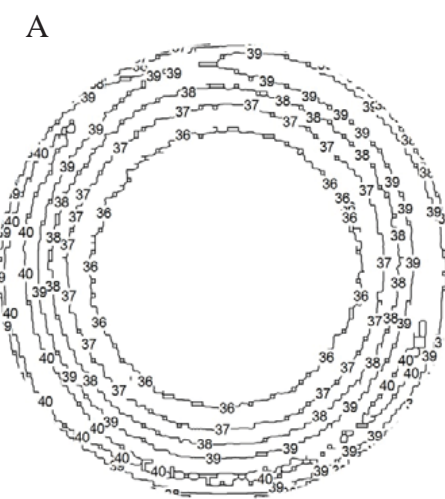

$\mathbf{C}$

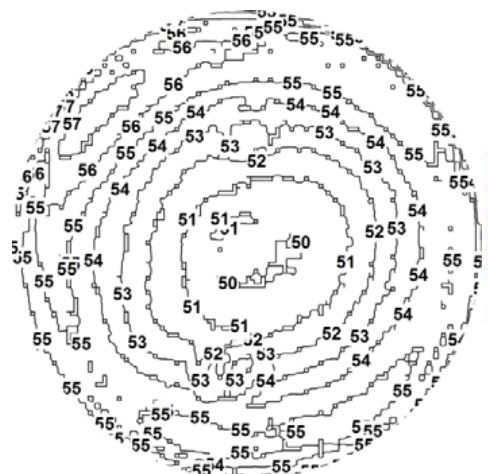

B

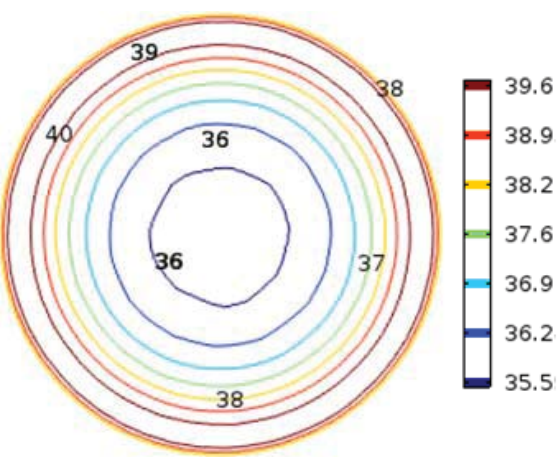

D

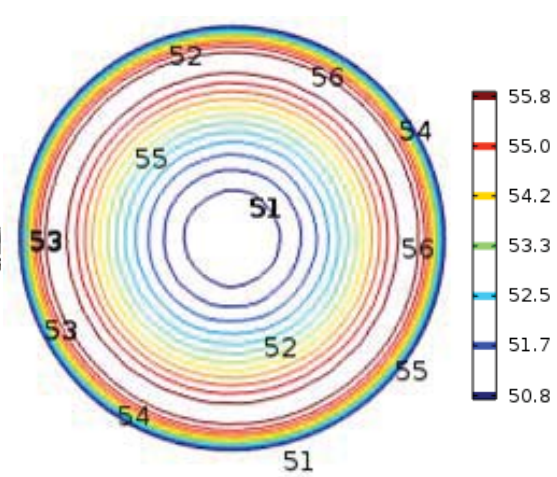

Fig. 1. Simulated and experimental temperature contour of the top surface of peanut butter (PB) and wheat flour (WF) treated by radio frequency with the electrode distances of $99 \mathrm{~mm}$ and $103 \mathrm{~mm}$, respectively. (A: temperature contour of PB from experimental result; B: temperature contour of PB from simulated data; C: temperature contour of WF from experimental result; D: temperature contour of WF from simulated data; unit: ${ }^{\circ} \mathrm{C}$ )

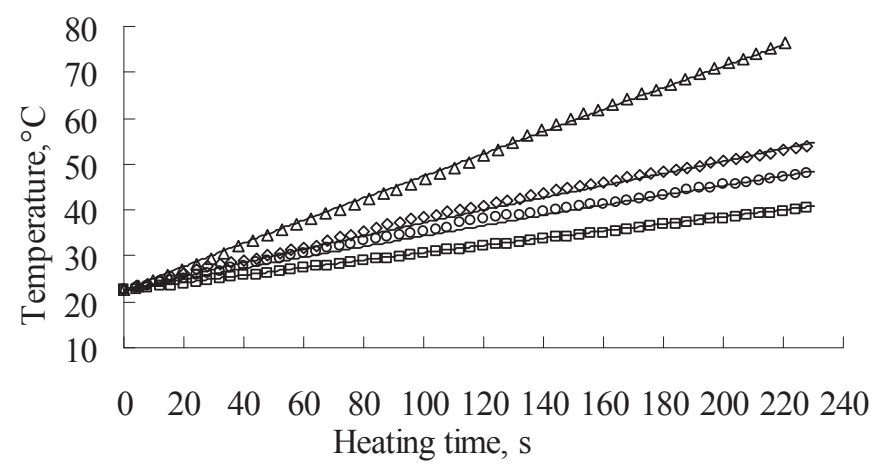

Fig. 2. Comparison of simulated and experimental results with time-temperature history in the physical centre point of peanut butter (PB) placed on the bottom electrode with the electrode distances of $84 \mathrm{~mm}(\Delta), 89 \mathrm{~mm}(\diamond)$, $93 \mathrm{~mm}(\circ)$, and $99 \mathrm{~mm}(\square)$ 


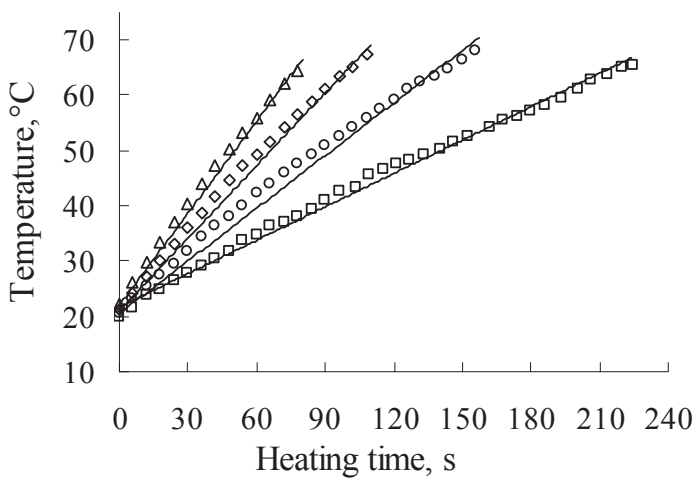

Fig. 3. Comparison of simulated and experimental results with time-temperature history in the physical centre point of wheat flour (WF) placed on the bottom electrode with the electrode gaps of $89 \mathrm{~mm}(\Delta), 93 \mathrm{~mm}(\diamond), 98$ $\mathrm{mm}(\circ)$, and $103 \mathrm{~mm}(\square)$

It should be noted that the cutting signs in the egde of Figure 1A and Figure 1C, which are temperatures of the sample and the container, are applied as the experimental data of infrared thermal imaging. But only the temperature distribution of the sample was selected and compared with those in Figure $1 \mathrm{~B}$ and Figure 1D, respectively. In Figure 1, the temperature distribution of RF heating of WF is more irregular than that of RF heating of PB, which is probably a consequence of the rapid heat dissipation of the porous material as well as its rough surface. However, it can identify the same trend of the temperature distribution as the simulated result.

\subsection{Research findings and discussion}

2.2.1. Effect of electrode distance on heating uniformity. The uniformity index (UI) of $\mathrm{PB}$ and WF under different electrode distances could be calculated by substituting the simulated 3D temperature into the Equation (4). According to Table 1, UI is negatively correlated with electrode distance. In other words, larger electrode distance will bring better heating uniformity and smaller heating rate. RF heating of $\mathrm{PB}$ achieves better uniformity than that of WF. This is caused by the larger air contact area and higher heat dissipation of powder materials. PB has a layer of oil film on the top surface, which reduces heat dissipation. Generally, UI of both PB and WF treated by RF heating is relatively large. Same with other foods, RF heating uniformity of PB and WF shall be improved by further studies.

2.2.2. Effect of electrode distance on heating rate. It can be seen from Figure 2 and Figure 3 that larger electrode distance will lead to smaller heating rate. Based on further study, a logarithmic linear relationship between electrode distance and heating rate was discovered (Fig. 4). The fitting equation is:

$$
\left\{\begin{array}{l}
x=\lg (d) \\
y=\lg (\tau) \\
y=a+b x
\end{array}\right.
$$

where $d$ is the electrode distance, $\mathrm{mm} ; \tau$ is heating rate, ${ }^{\circ} \mathrm{C} \mathrm{s}^{-1}, a$ and $b$ are coefficients. 
A

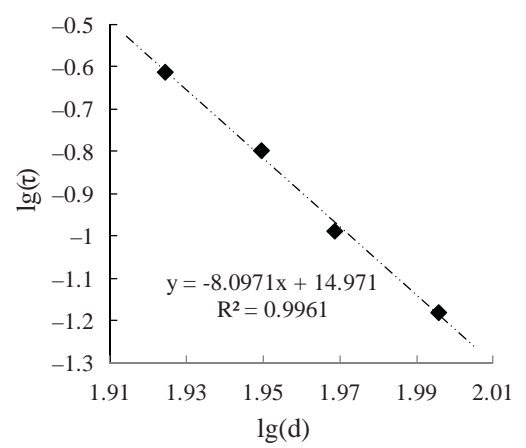

$\mathrm{C}$

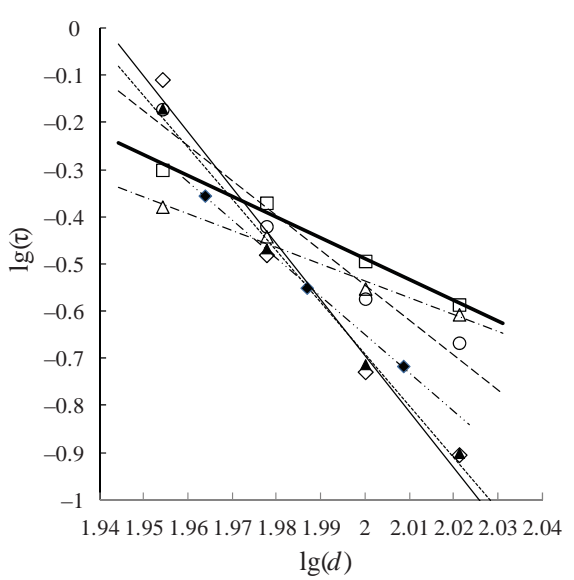

B

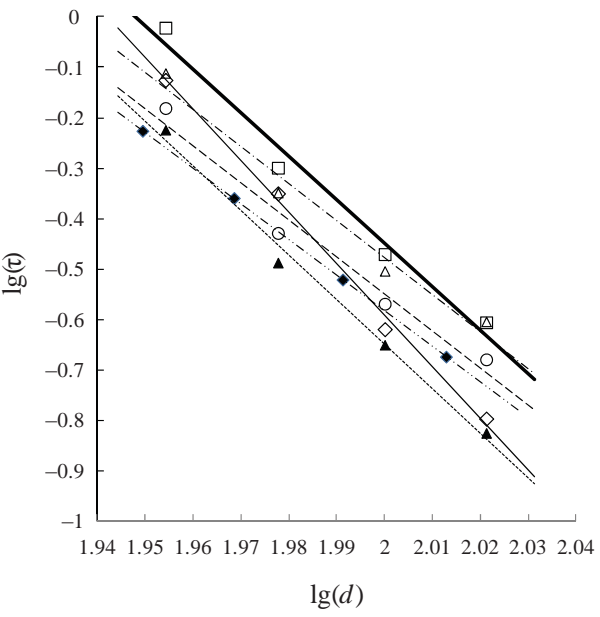

$\mathrm{D}$

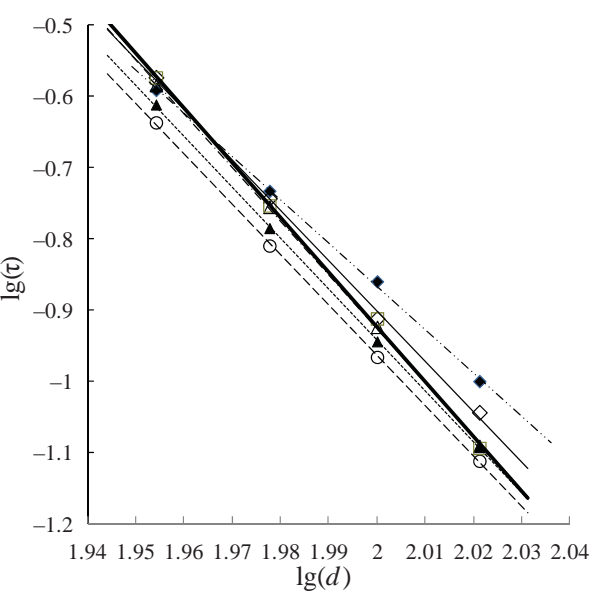

Fig. 4. Negative logarithmic linear relationship of electrode distance $\mathrm{d}(\mathrm{mm})$ and heating rate $\tau\left({ }^{\circ} \mathrm{C} \mathrm{s}^{-1}\right)$ for radio frequency (RF) heating of different type, shape, and size of materials. Cylinder I $(\diamond)$, its fitted curve is the double dot dash line; Cylinder II $(\diamond)$, its fitted curve is the thin solid line; Cube I $(\boldsymbol{\Delta})$, its fitted curve is the dotted line; Cube II ( $\Delta$ ), its fitted curve is the dashdot line; Cuboid I ( $\circ$ ), its fitted curve is the long dashed line; Cuboid II ( $\square$ ), its fitted curve is the heavy line (A: peanut butter (PB) ; B: plain flour; C: corn starch; D: milk powder)

Such negative logarithmic linear relationship was also found for other materials - corn starch and skimmed milk powder. This logarithmic linear relationship is unrelated to shape and size of materials. Figures 4B-D show three materials: plain flour, corn starch, and skimmed milk powder. Their shapes are cylinder I $(\Phi 100 \times 50 \mathrm{~mm})$, cylinder II $(\Phi 130 \times 60$ $\mathrm{mm})$, cube I $(88 \times 88 \times 52 \mathrm{~mm})$, cube II $(112 \times 112 \times 60 \mathrm{~mm})$, cuboid I $(167 \times 109 \times 56 \mathrm{~mm})$ and cuboid II $(197 \times 129 \times 64 \mathrm{~mm})$. There is a negative logarithmic linear relationship between electrode distance of RF heating system and heating rate. The fitting coefficients are shown in Table 2, which reflect high fitting accuracy. This not only is conducive to further 
study on RF heating efficiency, but also provides a heating rate value for calculation of electrode voltage and can be used in the calculation formula of electrode voltage (HuANG et al., 2015; JIAO et al., 2015).

Table 2. Fitting coefficients of the negative logarithmic linear relationship of electrode distance and heating rate (three kinds of material, six kinds of shape and size of samples)

\begin{tabular}{lccccccc}
\hline Materials & Coefficients & Cylinder I & Cylinder II & Cube I & Cube II & Cuboid I & Cuboid II \\
\hline \multirow{4}{*}{ Plain flour } & $a$ & 13.558 & 19.902 & 17.025 & 14.235 & 14.177 & 16.867 \\
& $b$ & -7.071 & -10.248 & -8.838 & -7.357 & -7.364 & -8.659 \\
& $\mathrm{R}^{2}$ & 1.000 & 0.996 & 0.991 & 0.974 & 0.972 & 0.979 \\
\multirow{5}{*}{ Corn starch } & $a$ & 15.486 & 23.000 & 21.189 & 6.596 & 14.213 & 8.282 \\
& $b$ & -8.068 & -11.847 & -10.940 & -3.566 & -7.379 & -4.385 \\
& $\mathrm{R}^{2}$ & 0.999 & 0.980 & 0.994 & 0.983 & 0.966 & 0.987 \\
\multirow{5}{*}{ Milk powder } & $a$ & 11.263 & 13.276 & 13.385 & 14.201 & 13.227 & 14.483 \\
& $b$ & -6.065 & -7.088 & -7.163 & -7.564 & -7.096 & -7.703 \\
& $\mathrm{R}$ & 0.999 & 0.999 & 1.000 & 1.000 & 1.000 & 0.999 \\
\hline
\end{tabular}

2.2.3. Discussion of the findings. Electrode distance is one of the important parameters in RF heating of foods and agricultural products, while the heating rate and the heating uniformity are two important indices. The influence of electrode distances on the heating behaviours can be explained by the Dielectric Polarization Theory.

A high-frequency AC electromagnetic field will be generated between the upper and bottom electrodes during the operation of RF system, resulting in changes of interior microscopic particles in materials. Positive and negative charge centres of non-polar particles suffer relative displacement and the molecule enters an induced dipole state; the dipole of polar molecule rotates with the external electric field; positive and negative ions move along corresponding electric field directions and generate ionic polarization; bound charges in regular arrangement occur on the dielectric surface, which is perpendicular to the external electric field. The positive and negative charges of the dipole move toward the opposite direction when the external electric field inverts. During this process, friction-like effects are generated due to interaction of adjacent particles and "thermal motion" of particles, which makes dielectric medium gain energy, manifested by increasing temperature. This phenomenon intensifies with the increase of frequency or intensity of the external electric field. The electric field intensity decreases when the electrode distance increases. According to Figure 5 and Table 3, the electric polarization and range (difference between the maximum and minimum values) decrease, thus decreasing the heating rate and heating uniformity accordingly.

Since the RF heating mechanism of foods and agricultural products is still unclear, the negative logarithmic linear relationship between the electrode distance and the heating rate has not been discussed theoretically in this paper. 

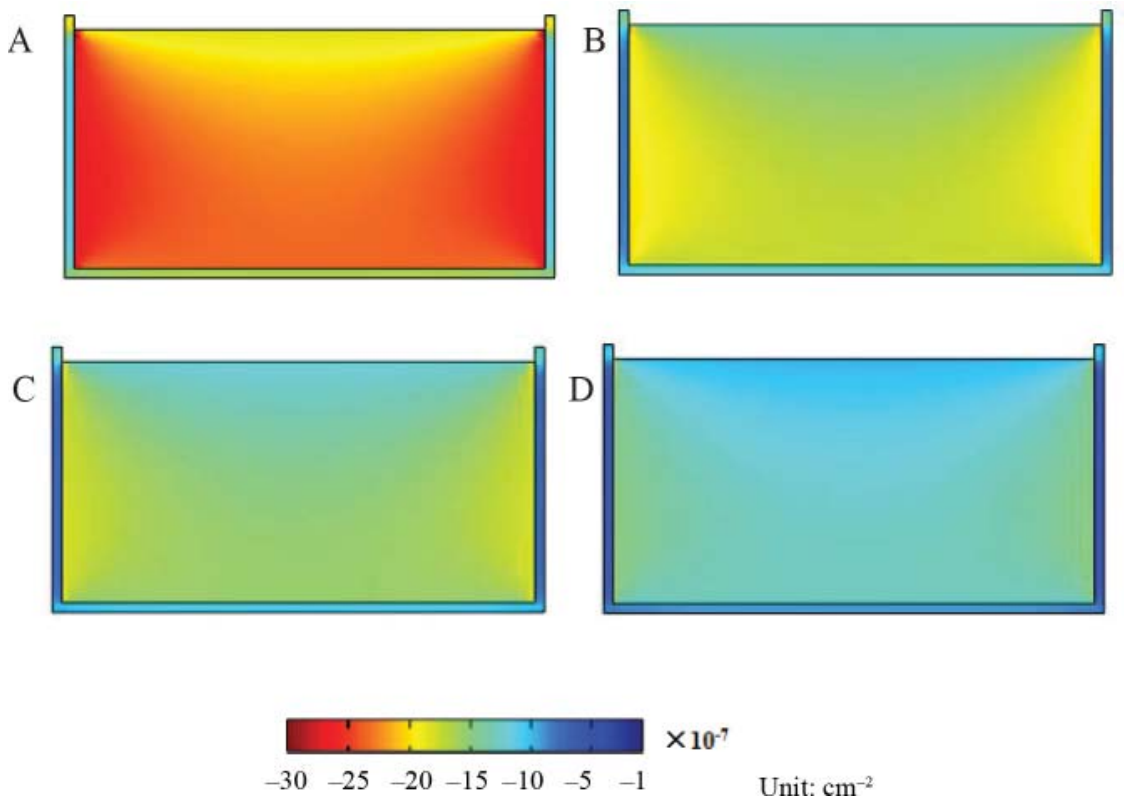

Fig. 5. Electric polarization distribution of peanut butter sample related to different electrode distances (A: $84 \mathrm{~mm}$; B: $89 \mathrm{~mm}$; C: $93 \mathrm{~mm}$; : $99 \mathrm{~mm}$ )

Table 3. Electric polarization values (minimum, maximum, average, and range) of sample related to different electrode distances. Unit: $\mathrm{cm}^{-2}$

\begin{tabular}{lcccc}
\hline$\times 10^{-7}$ & $\mathrm{~d} 84$ & $\mathrm{~d} 89$ & $\mathrm{~d} 93$ & $\mathrm{~d} 99$ \\
\hline Minimum & -20.40 & -19.81 & -18.54 & -17.45 \\
Maximum & -12.39 & -11.35 & -10.50 & -9.79 \\
Average & -16.25 & -15.10 & -14.12 & -13.27 \\
Range & 8.89 & 8.46 & 8.05 & 7.67 \\
\hline
\end{tabular}

\section{Conclusions}

In this study, radio frequency (RF) heating uniformity and heating rate of peanut butter (PB) (high-protein oily thick food) under four electrode distances $(84 \mathrm{~mm}, 89 \mathrm{~mm}, 93 \mathrm{~mm}$, and 99 $\mathrm{mm}$ ) and wheat flour (WF) (powder food) under four different electrode distances $(89 \mathrm{~mm}$, $93 \mathrm{~mm}, 98 \mathrm{~mm}$, and $103 \mathrm{~mm}$ ) were analysed by the numerical simulation model of RF heating of low moisture foods based on electromagnetic-thermal field coupling. Some conclusions gained:

(i) The results of the numerical simulation model agree with experimental results.

(ii) Uniformity index $(U I)$ is negatively correlated with electrode distance. In other words, larger electrode distance will contribute to better heating uniformity. RF heating of low moisture foods has poor heating uniformity. Further research on the improvement of heating uniformity is needed.

(iii) There is a negative logarithmic linear relationship between electrode distance of the RF system and heating rate. Such relationship is unrelated to type, shape, and size of materials. 
This is not only conducive to further study on RF heating efficiency, but also provides a heating rate value for calculation of electrode voltage.

This research was supported by grant (No. 2015J01184) from Natural Science Foundation of the Department of Science \& Technology of Fujian Province, China.

\section{References}

Boreddy, S.R., Birla, S.L., Froning, G., Thippareddi, H. \& Subbiah, J. (2014): Effect of radio frequency assisted thermal processing on quality and functional properties of egg white powder. Trans. ASABE, 57(6), 17611770.

Chan, T.C., TAng, J. \& Younce, F. (2004): 3-Dimensional numerical modeling of an industrial radio frequency heating system using finite elements. J. Microwave Power EE., 39(2), 87-105.

Choi, С.T. M. \& KonRAD, А. (1991): Finite element modeling of the RF heating process. IEEE Trans. Magn., 27(5), 4227-4230.

Gevere, D.J., Bigley, A.B. \& Brunkhorst, C.D. (2017): Pasteurization of shell eggs using radio frequency heating. J. Food Eng., 193, 53-57.

Huang, Z., Zhu, H., YAn, R. \& WANG, S. (2015): Simulation and prediction of radio frequency heating in dry soybeans. Biosyst. Eng., 129, 34-47.

JIAO, Y., TANG, J. \& WANG, S. (2014a): A new strategy to improve heating uniformity of low moisture foods in radio frequency treatment for pathogen control. J. Food Eng., 141, 128-138.

JiAO, Y., TANG, J., Wang, S. \& Koral, T. (2014b): Influence of dielectric properties on the heating rate in freerunning oscillator radio frequency systems. J. Food Eng., 120, 197-203.

JiAO, Y., SHI, H., TANG, J., LI, F. \& WANG, S. (2015): Improvement of radio frequency (RF) heating uniformity on low moisture foods with polyetherimide (PEI) blocks. Food Res. Int., 74, 106-114.

JiAo, S., Zhong, Y. \& Deng, Y. (2016a): Hot air-assisted radio frequency heating effects on wheat and corn seeds: Quality change and fungi inhibition. J. Stored Prod. Res., 69, 265-271.

JiAo, S., Zhu, D., Deng, Y. \& Zhaо, Y. (2016b): Effects of hot air-assisted radio frequency heating on quality and shelf-life of roasted peanuts. Food Bioprocess Tech., 9(2), 308-319.

Kong, L., Zhang, M., WANG, Y., AdHIKARI, B. \& YANG, Z. (2016): Evaluation of heating uniformity in radio frequency heating systems using carrot and radish. Int. Agrophys., 30(4), 465-473.

Metaxas, A.C. (1996): Foundations of electroheat - A unified approach. Wiley, 530 pages. -ref: Fuel and Energy Abstracts, 37(3), 193.

Ozturk, S., Kong, F., Trabelsi, S. \& Singh, R.K. (2016): Dielectric properties of dried vegetable powders and their temperature profile during radio frequency heating. J. Food Eng., 169, 91-100.

PAN, L., JiAO, S., GAUTZ, L., Tu, K. \& WANG, S. (2012): Coffee bean heating uniformity and quality as influenced by radio frequency treatments for postharvest disinfestations. Trans. ASABE, 55(6), 2293-2300.

ShI, H., JiAo, Y., TANG, J., Zhang, S., HE, J. \& KuANG, P. (2016): Heating uniformity evaluation and improvement of radio frequency treated pre-packaged food. Trans. ASABE, 59(5), 1441-1450

Tiwari, G., Wang, S., TANG, J. \& BirLA, S.L. (2011): Computer simulation model development and validation for radio frequency (RF) heating of dry food materials. J. Food Eng., 105(1), 48-55.

Uyar, R., Erdogdu, F., SARghini, F. \& Marra, F. (2016): Computer simulation of radio-frequency heating applied to block-shaped foods: Analysis on the role of geometrical parameters. Food Bioprod. Process., 98, 310-319.

WANG, S., Yue, J., TANG, J. \& Chen, B. (2005): Mathematical modelling of heating uniformity for in-shell walnuts subjected to radio frequency treatments with intermittent stirrings. Postharvest Biol. Tec., 35(1), 97-107.

WANG, J., OlsEn, R.G., TANG, J. \& TANG, Z. (2007): Influence of mashed potato dielectric properties and circulating water electric conductivity on radio frequency heating at $27 \mathrm{MHz}$. J. Microwave Power EE., 42(2), 31-46.

WAng, S., Luechapattanaporn, K. \& TAng, J. (2008): Experimental methods for evaluating heating uniformity in radio frequency systems. Biosyst. Eng., 100(1), 58-65.

Wang, S., TANG, J., Johnson, J.A. \& CAVALIERI, R.P. (2013): Heating uniformity and differential heating of insects in almonds associated with radio frequency energy. J. Stored Prod. Res., 55, 15-20.

WANG, Y., ZhANG, L., GAO, M., TANG, J. \& WANG, S. (2014): Evaluating radio frequency heating uniformity using polyurethane foams. J. Food Eng., 136, 28-33. 